\title{
Effect of Chloroquine on Morphology of Cytoplasmic Granules in Maturing Human Leukocytes-an Ultrastructural Study*
}

\author{
Martha Fedorko \\ (From The Rockefeller University, New York)
}

\begin{abstract}
Bone marrow and peripheral blood from patients who had received chloroquine phosphate were studied to determine the effect of this drug on the ultrastructure of cytoplasmic granules in leukocytes. Neutrophils from approximately one-half of the patients who were treated developed abnormal cytoplasmic granules. Vacuolar, lamellar, and particulate components within abnormal, large granules were present in myelocytes from certain patients who received chloroquine therapy. Mature neutrophils and lymphocytes from these patients showed variable numbers of large, membrane-bounded structures containing myelin figures. Cytoplasmic granules in eosinophilic myelocytes from patients treated with chloroquine did not contain the usual crystalloid structure, but instead contained small whorls of osmiophilic material. The granules in abnormal mature eosinophils were replaced by large vacuoles which contained amorphous material. The abnormal granules seen in these various white cells after chloroquine therapy may either reflect defective granule formation or autophagy.
\end{abstract}

\section{Introduction}

Previous work by others has helped to establish the true nature of cytoplasmic granules normally found within leukocytes. Extensive biochemical and functional studies have demonstrated that such cytoplasmic granules are lysosomes $(1,2)$.

Although stained smears of human leukocytes have been studied for approximately 100 years, very little is known about abnormal leukocyte granulation. Abnormal granules in human leukocytes have been observed in both hereditary and acquired disorders. In the case of the latter, the etiology is diverse and the leukocyte abnormality is often reversible. In preparations stained by the Wright-Giemsa method normal human neutrophil granules are pink-to-purple structures approx-

* Received for publication 5 June 1967 and in revised form 24 July 1967.

This work was supported by grants AI 01831 and FR 00102 from the U. S. Public Health Service, National Institutes of Health, Bethesda, Md.

Address requests for reprints to Dr. M. Fedorko, The Rockefeller University, 66 Street and York Avenue, New York, N. Y. 10021. imately $0.3 \mu$ in diameter, whereas the so-called "toxic" granules appear as $0.5-1 \mu$ basophilic granules. The ultrastructure of any type of abnormal acquired leukocyte granulation has not been described and the pathophysiology of the changes is unknown.

During the course of a study on human marrow, abnormal, large basophilic granules in neutrophilic leukocytes were noted on a smear from a patient who received a course of chloroquine phosphate, 7-chloro-4- (4-diethylamino-1 -methylbutylamino) quinoline phosphate, therapy for sarcoidosis. "Toxic" granules have been noted previously on blood smears from patients who have been treated with chloroquine (3). This communication will describe the ultrastructural changes in marrow and peripheral blood leukocytes from patients who received chloroquine phosphate therapy.

\section{Methods and Materials}

Administration of drug. Six patients with sarcoidosis were given $250-500 \mathrm{mg}$ chloroquine phosphate (Winthrop Laboratories, N. Y.) orally, daily for periods of 2-6 months. Diagnosis was confirmed in all patients in 
the study by positive Kveim tests and clinical history compatible with sarcoidosis.

Six patients with sarcoidosis who received no therapy were also studied.

Preparation of tissue specimens. Serial samples of peripheral blood were collected from all patients before, during, and after treatment with chloroquine and were prepared for electron microscopy in the following manner. Blood was collected into heparinized capillary tubes (Yankee, Clay-Adams, N. Y.) and centrifuged at $2500 \mathrm{~g}$ for $15 \mathrm{~min}$. The glass capillary tube was broken above the buffy coat layer; the buffy coat was then expressed into fixative.

Bone marrow was aspirated from the posterior iliac crest of one patient during and 2 months after a course of chloroquine therapy. The marrow was collected into a syringe coated with normal saline which contained heparin (Panheparin, Abbot, Chicago, Ill.) in a final concentration of $1.5 \mathrm{mg} / \mathrm{ml}$. The marrow suspension was then centrifuged at $250 \mathrm{~g}$ for $5 \mathrm{~min}$. Small particles of marrow which contained fat rose to the top of the plasma, were aspirated, and then were either prepared for light microscopy or fixed for electron microscopy.

Light microscopy. Smears of both peripheral blood and bone marrow particles were air-dried and stained with Wright-Giemsa stain.

Fixation for clectron microscopy. Specimens were either fixed $1 \mathrm{hr}$ at room temperature or overnight at $4^{\circ} \mathrm{C}$ in $2.5 \%$ glutaraldehyde in $0.1 \mathrm{M}$ phosphate buffer $\mathrm{pH}$ 7.4 , made by diluting $1: 20$ a stock solution of $50 \%$ glutaraldehyde (Fischer Scientific Company, Pittsburgh, $\mathrm{Pa}$.) The specimens were then washed twice in cold isotonic saline for $30 \mathrm{~min}$ and postfixed in $1 \%$ osmium tetroxide in $0.1 \mathrm{M}$ phosphate buffer for $1 \mathrm{hr}$ in the cold. Blocks were washed twice in cold isotonic saline before being dehydrated in graded alcohols and embedded in Epon (4). The micrographs which appear in this paper are from cells which have been fixed by the above method. Some specimens were also fixed in $1 \%$ osmium tetroxide in $0.1 \mathrm{M}$ phosphate buffer $\mathrm{pH} 7.4$ for $1 \mathrm{hr}$ at $4^{\circ} \mathrm{C}$ and then received three $15-\mathrm{min}$ washes in cold isotonic saline before dehydration and embedding.

Electron microscopic localization of acid phosphatase. $10 \mathrm{ml}$ of heparinized blood was sedimented for $1 \mathrm{hr}$. The white cell-rich plasma was separated off and centrifuged at $400 \mathrm{~g}$ for $5 \mathrm{~min}$. The cell pellet was fixed by suspending the cells for $15 \mathrm{~min}$ in $1.25 \%$ glutaraldehyde in cacodylate buffer, washed twice in 0.25 M sucrose, and then incubated for $1 \mathrm{hr}$ at $37^{\circ} \mathrm{C}$ in $5 \mathrm{ml}$ of prewarmed Gomori substrate $\mathrm{pH} 5.0$, which had been freshly prepared with $\beta$-glycerophosphate (Eastman Kodak, Rochester, N. Y.) The cells were then washed once and postfixed for $30 \mathrm{~min}$ in $1 \%$ osmium in cacodylate buffer. After osmium fixation the cell pellet was washed twice for $30 \mathrm{~min}$ in cold saline before dehydration and embedding. Cells incubated with inhibitors or without substrate failed to show reactivity.

All blocks were sectioned on a Porter Blum microtome with a diamond knife, stained with uranyl acetate and lead citrate (5), and examined in a Siemens Elmiskop I.

\section{Results}

Abnormal granules in peripheral blood leukocytes occurred in three out of seven patients with sarcoidosis who received $250-500 \mathrm{mg}$ of oral chloroquine phosphate daily for 2-6 months. Seven patients with sarcoidosis who received no therapy did not exhibit any abnormality in leukocytes. In one patient the ultrastructural changes were present in peripheral blood and bone marrow leukocytes during two successive 3-month courses of chloroquine therapy. After cessation of chloroquine therapy the numbers of abnormal cells in peripheral blood progressively diminished. 1 month after the drug was discontinued, the ultrastructure of all leukocytes in blood and bone marrow was normal. During the course of therapy the white blood cell count in the peripheral blood of one patient decreased from 5200 to 2800 and became normal after cessation of therapy. The other two patients had normal blood counts during therapy. The patients who were receiving cholorquine phosphate and who demonstrated cytologic changes in leukocytes were not observed to have increased numbers of infections, gastrointestinal distress, or difficulties with vision.

Neutrophils. Mature neutrophils from blood or bone marrow of sarcoidosis patients who had received no therapy were identical in ultrastructure to cells obtained from normal persons (Fig. 1). The cytoplasmic granules appeared as round, oblong, or biconcave electron-opaque structures which varied in size and shape. They were membrane bounded and for the most part did not possess any internal structure, but occasionally contained a crystalloid inclusion. Variable amounts of glycogen, sparse amounts of rough endoplasmic reticulum, and occasional mitochondria were present in the cytoplasm. On a stained smear the mature cells showed typical small $0.2-0.5 \mu$ pinkto-purple granules (see insert, Fig. 1). The ultrastructure of an abnormal mature neutrophil from peripheral blood of a patient treated with chloroquine is seen in Fig. 2. Many of the cytoplasmic granules were normal in appearance but there was striking alteration in the ultrastructure of other cytoplasmic granules. The abnormal granules were larger than the normal ones, and they presented an internal structure of layered membranous elements (myelin figures). These myelin 
figures were also observed after the fixation with osmium alone. Identical changes were seen in mature neutrophils from bone marrow. Mature circulating neutrophils contained occasional, large abnormal granules which were positive for acid phosphatase after enzyme histochemistry was per- formed at the ultrastructural level. The increased size and intense staining of the cytoplasmic granules on a smear is demonstrated in the insert in Fig. 2.

Bone marrow myelocytes from sarcoidosis patients who did not receive chloroquine and from

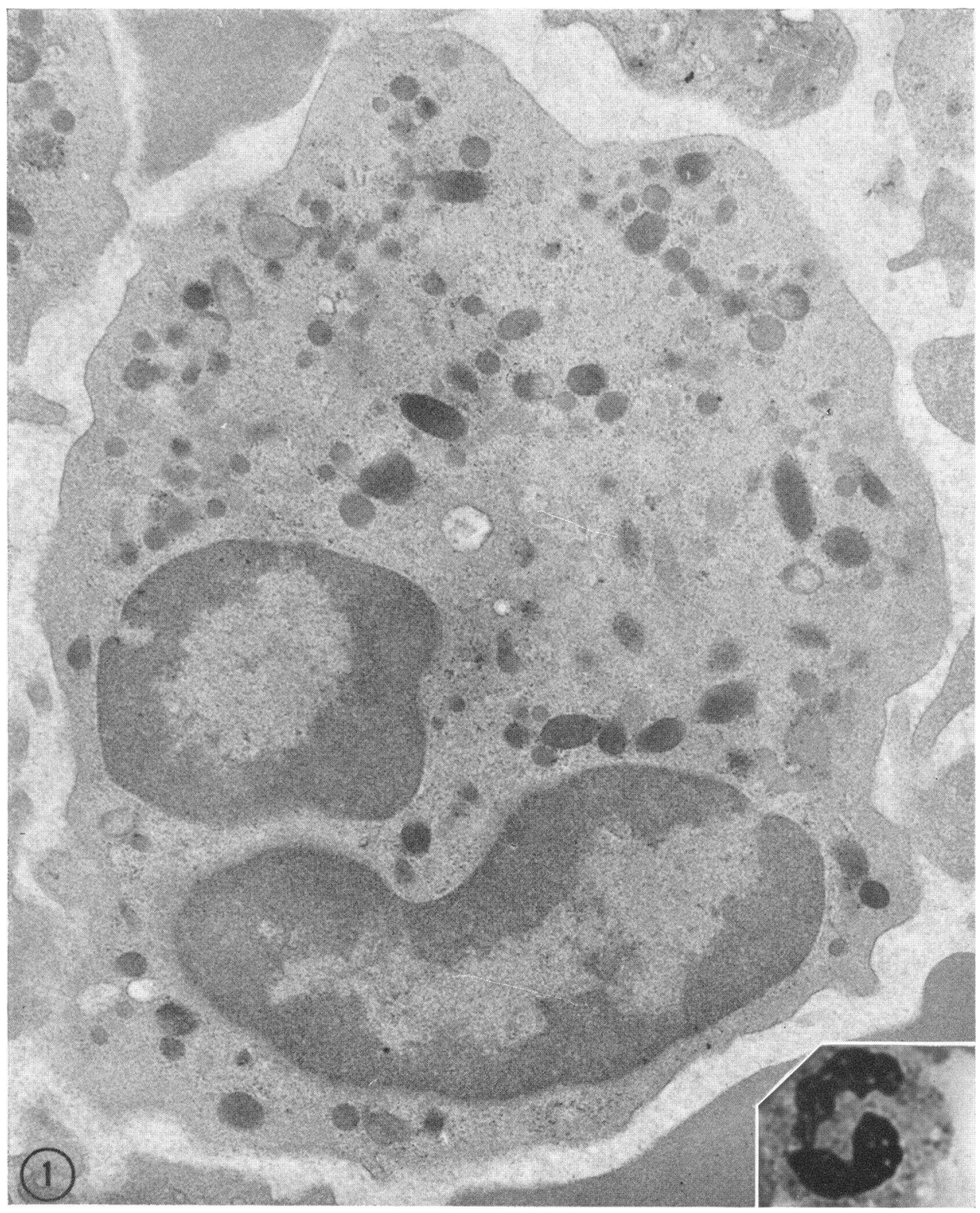

Fig. 1. Mature neUtrophil from a patient With sarcoidosis Who received No therapy. Shown are two lobes of the nucleus and abundant cytoplasmic granules which vary in size and shape. The appearance is identical with that of neutrophils from normal persons. $\times 21,000$. The inset depicts the light microscopic appearance of a normal human neutrophil which was stained by the Wright-Giemsa method. The lobulated nucleus and pale-staining granules are seen. $\times 1900$. 


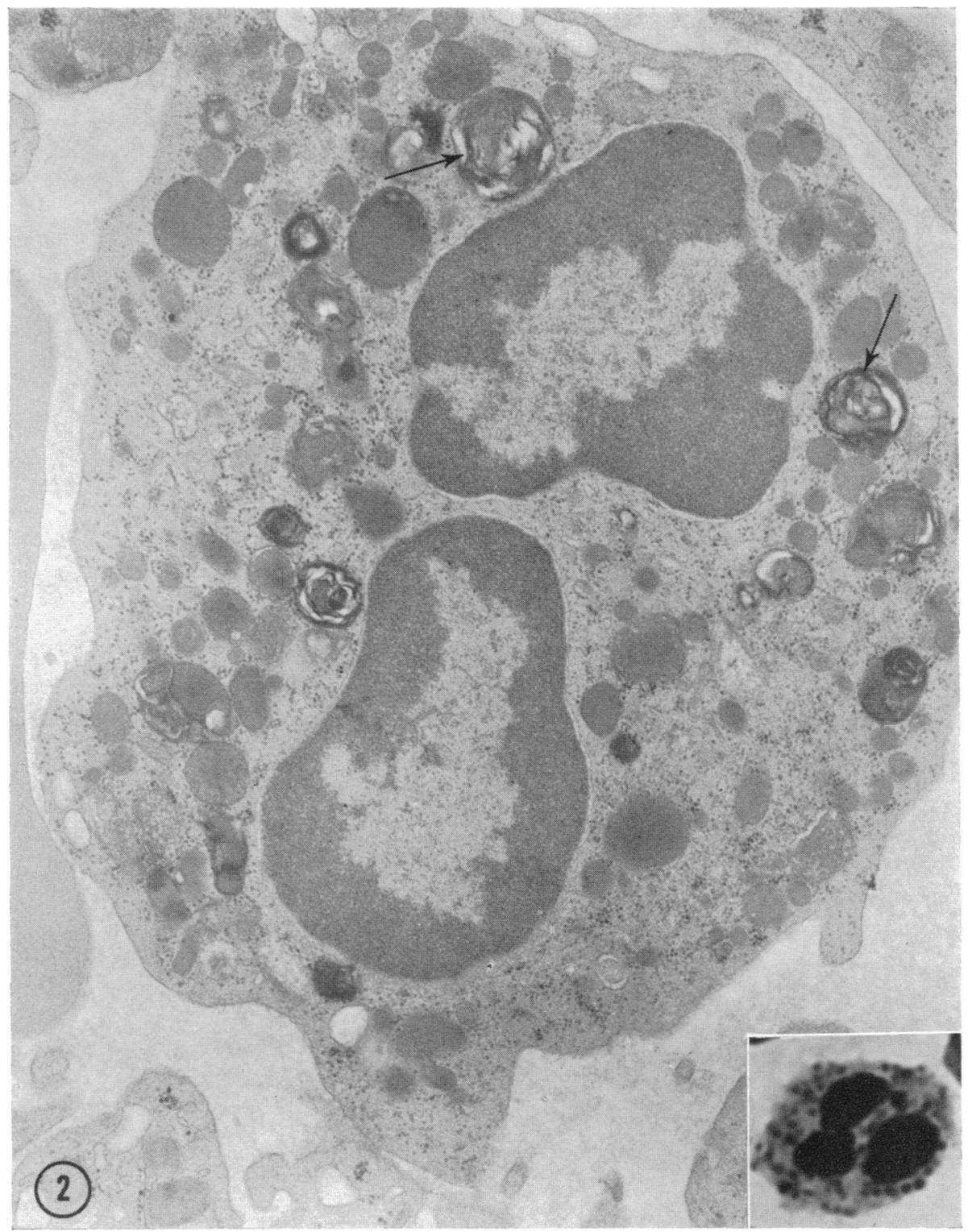

Fig. 2. Mature NeUtrophil from a patient With Sarcoidosis Who Was Receiving CHLOROQUINE PHOSPHATE THERAPY. The cytoplasm shows many large granules containing myelin figures (arrows). Apparently normal granules are also present. $\times 22,000$. The inset depicts the light microscopic appearance of a neutrophil from a patient during treatment with chloroquine phosphate. Lobes of the nucleus are visible as well as the large, intensely staining cytoplasmic granules. Method of preparation and stain is identical with that in Fig. 2. $\times 1900$.

normal persons contained membrane-bounded cytoplasmic granules which did not have any internal structure (Fig. 3). Smooth and rough endoplasmic reticulum, often showing dilated cisternae, was widely distributed in the cytoplasm. The Golgi complex occupied a relatively small area in human myelocytes; vesicular, vacuolar, and lamellar elements were seen. A myelocyte from a patient who was receiving chloroquine therapy is shown in Fig. 4. The affected granules were abnormally large and contained internal structure which consisted of vacuolar, lamellar, and par- 


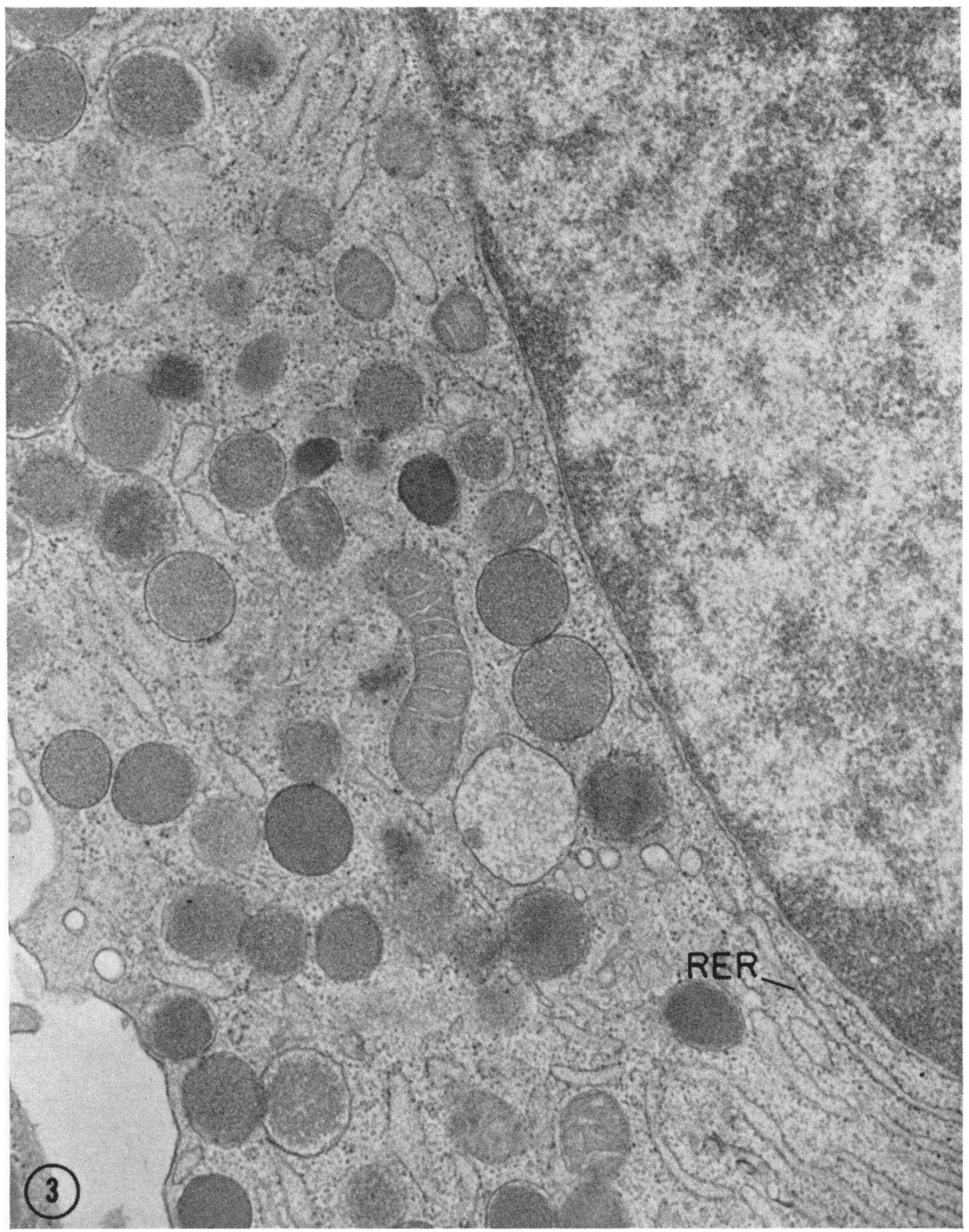

Fig. 3. NoRmal hUman myelocyte. There are cytoplasmic granules which are membrane bounded and without inclusions. Scattered elements of rough and smooth endoplasmic reticulum $(R E R)$ are present in the cytoplasm. $\times 33,000$.

ticulate inclusions. In some instances these components appeared fused to the periphery of the abnormal granule. Another abnormal myelocyte is shown in Fig. 5. Several granules had incorporated vacuolar elements and there was some evidence of early myelin figure formation.

Eosinophils. Normal mature eosinophils from untreated patients with sarcoidosis or from normal persons contained cytoplasmic granules with the characteristic internal crystalloid (6). Normal eosinophilic myelocytes contained two types of granules: those without any internal structure and those with a crystalloid inclusion.

The ultrastructure of mature, circulating eosinophils from certain sarcoidosis patients treated with chloroquine was profoundly affected. The abnor- 


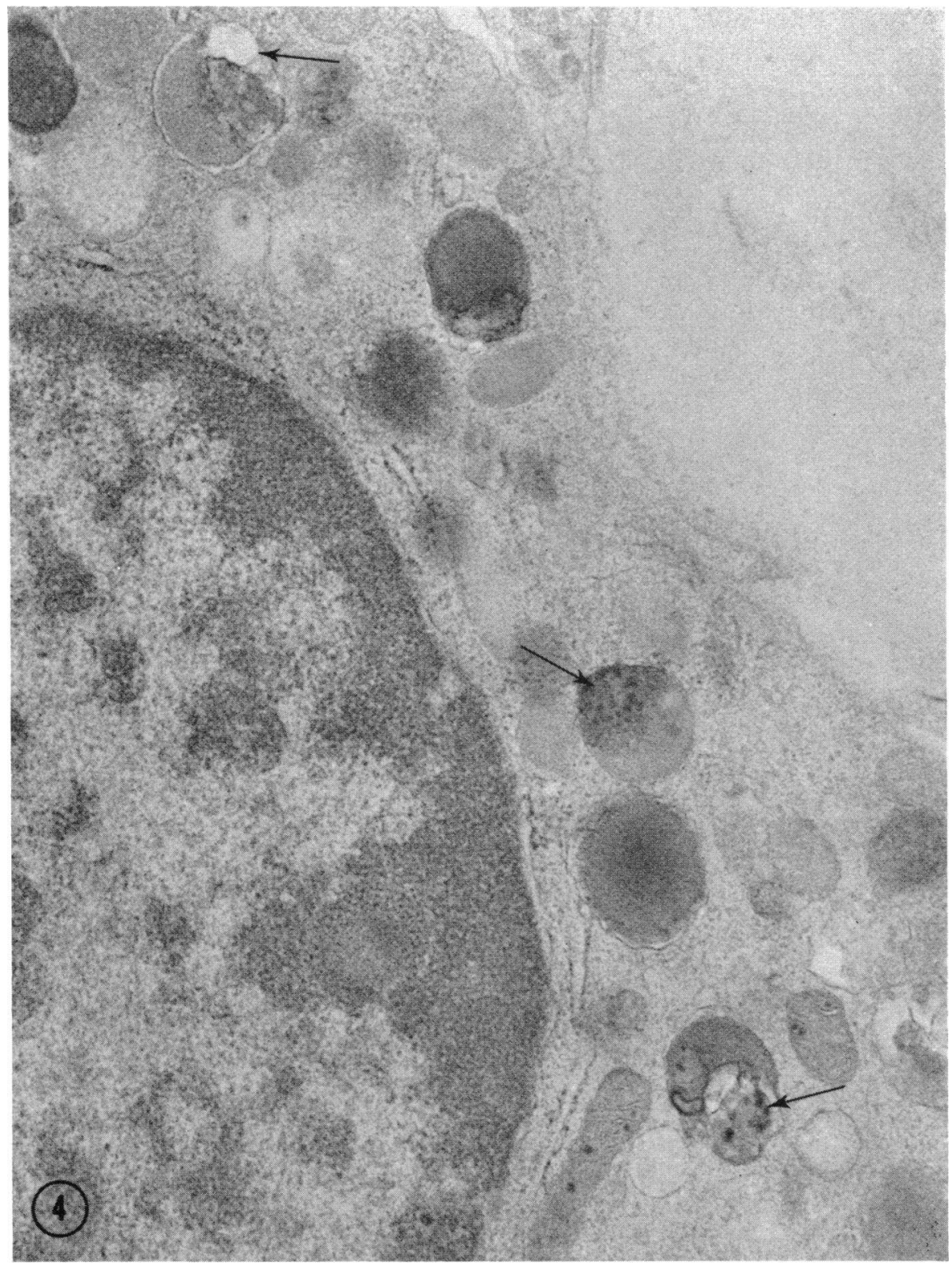

Fig. 4. Myelocyte from patient with SARCoidosis who Was treated With chloroQUINE. Note the numerous cytoplasmic granules (arrows) which contain particulate, lamellar, and vacuolar components. $\times 35,000$.

mal eosinophils showed many vacuoles containing amorphous material and only rare normal granules containing the characteristic internal crystalloid. The abnormal vacuoles in mature eosinophils were not positive for acid phosphatase. Eosinophilic myelocytes from patients receiving chloroquine showed in addition to some normal granules without any internal structure abnormal granules which contained osmiophilic whorls of material (Fig. 6) and dilated rough endoplasmic reticulum. The appearance of eosinophils from treated and untreated patients was normal on stained smears.

Mononuclear cells. The lymphocytes in bone marrow and peripheral blood from patients treated with chloroquine also showed ultrastructural changes. The abnormal cells contained membrane- 


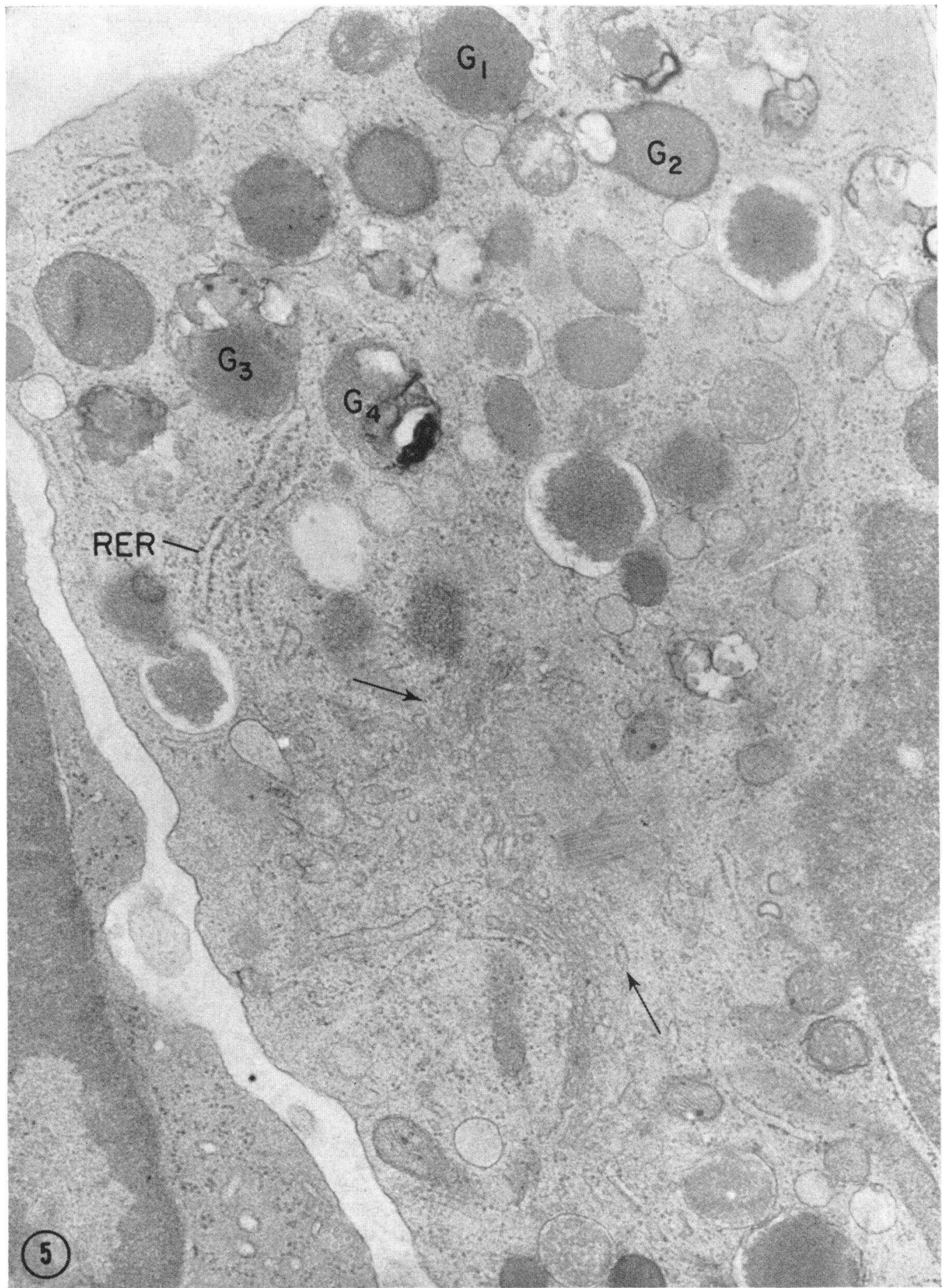

Fig. 5. Bone marrow myelocyte from a Patient who was ReCeiving chloroguine. The Golgi complex (arrows) and scattered elements of rough endoplasmic reticulum $(R E R)$ are present. Granules $\left(G_{1}\right)$ and $\left(G_{s}\right)$ appear to contain vacuolar components. Granule $G_{s}$ contains particulate elements, whereas $G_{4}$ appears to be composed of lamellae. $\times 35,000$. 


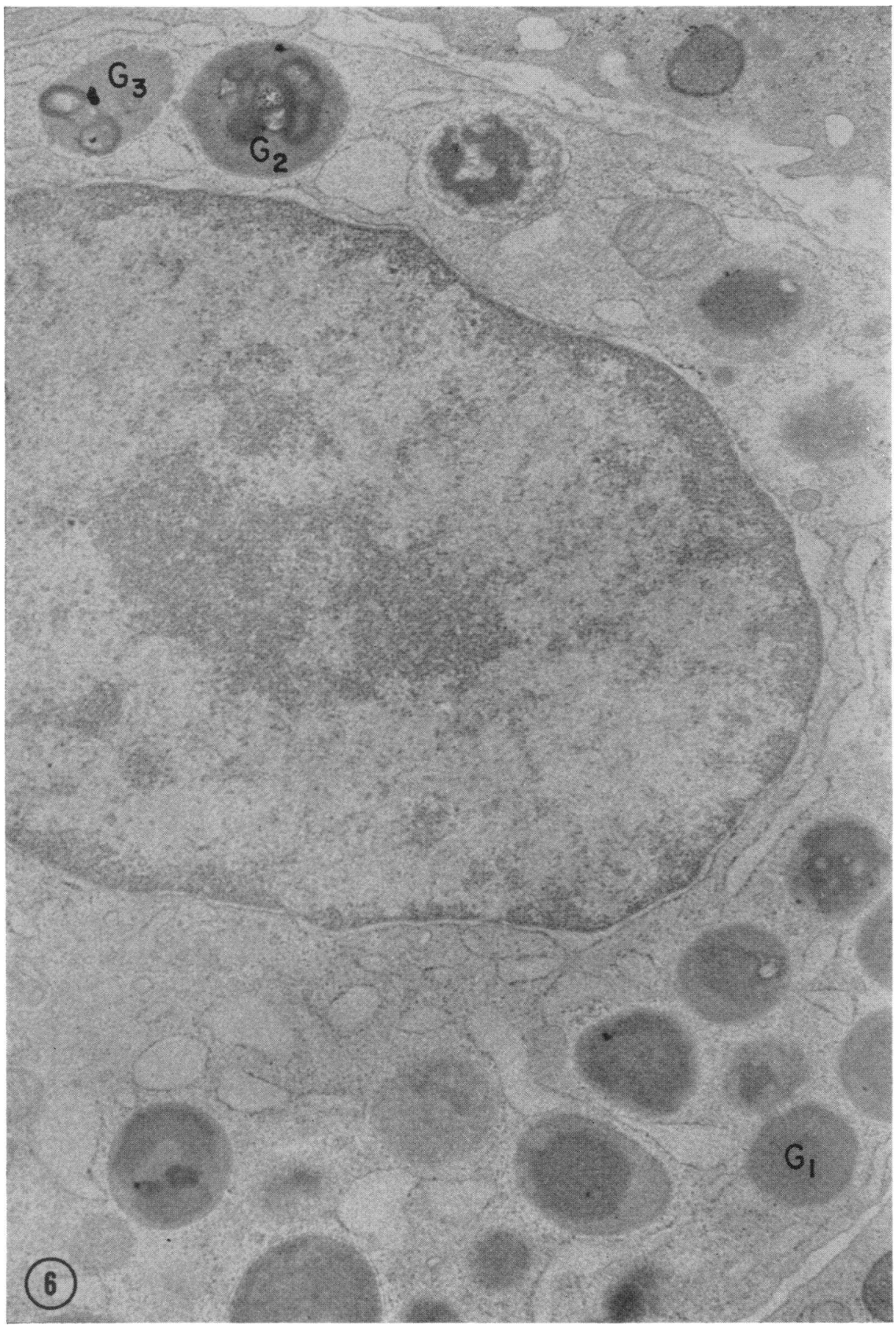

Fig. 6. EOSINOPHILIC MYELOCYTE FROM BONE MARROW OF A PATIENT WHO WAS RECEIVING CHLOROQUINE THERAPY. Normal cytoplasmic granules show either a crystalloid inclusion (not illustrated) or a homogenous dense matrix $\left(G_{1}\right)$, whereas the abnormal granules contain dense osmiophilic whorls $\left(G_{2-s}\right) . \times 27,000$. 


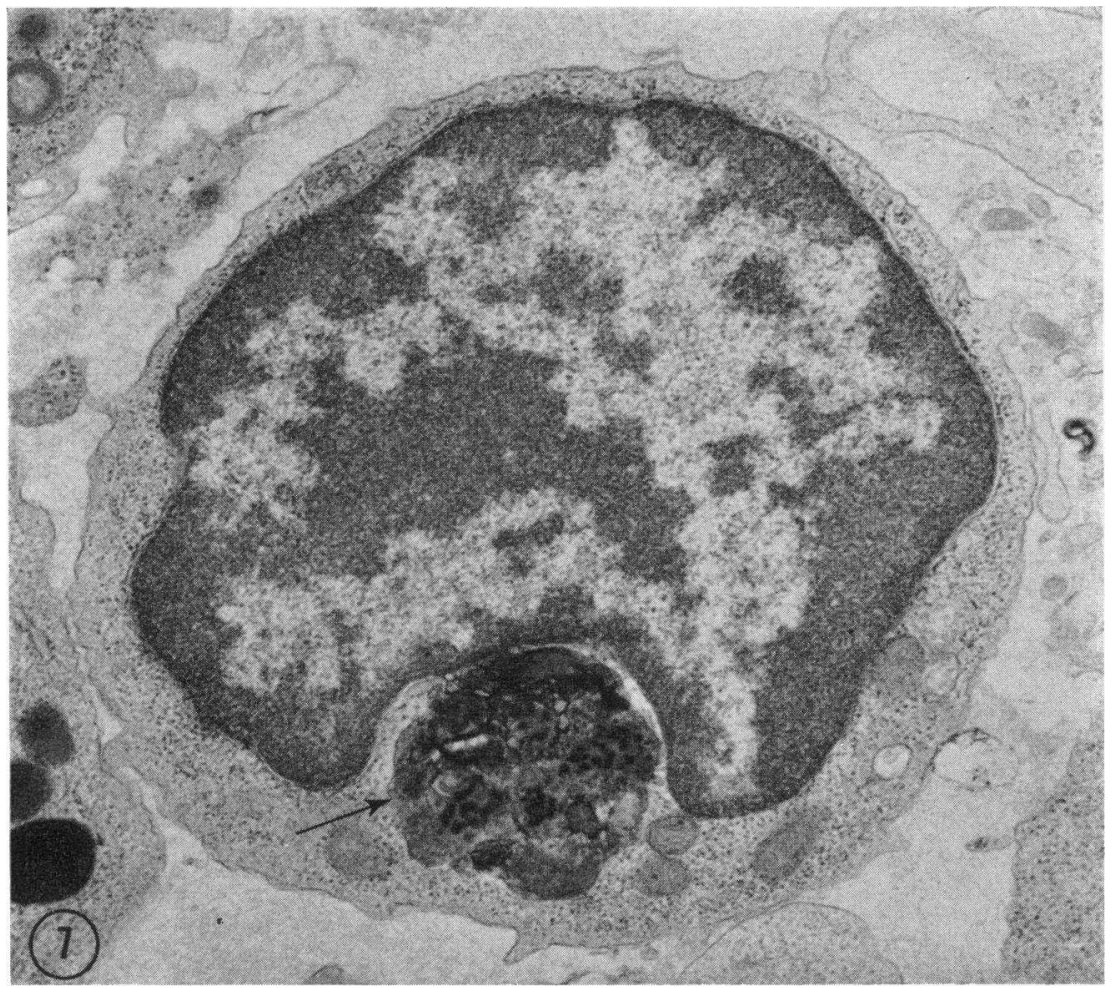

Fig. 7. Lymphocyte from a patient Who WAS RECEIVING ChloRoQUiNe phosPHATE THERAPY. A large membrane-bounded inclusion (arrow) which is composed of lamellar and particulate components is present within the cytoplasm. $\times 23,200$.

bounded myelin figures of various sizes. Some of the abnormal cytoplasmic structures were quite large (Fig. 7). These structures were positive for acid phosphatase. Except for these cytoplasmic inclusions, ultrastructural characteristics were those commonly found in lymphocytes. Lymphocytes in smears of blood and bone marrow from these patients contained occasional azurophilic granules. Evaluation of structural changes in histiocytes and monocytes was not included in this study because of the difficulty in identifying their precursors in bone marrow specimens on the basis of morphology alone.

\section{Discussion}

The results presented here demonstrate that chloroquine phosphate can exert a profound effect on cytoplasmic granules in immature and mature human leukocytes. The ultrastructure of acquired abnormal leukocyte granulation produced by this drug has been described. Previous work by others would seem to indicate that chloroquine is incorporated into leukocytes (3). The ultrastructural changes are probably, therefore, a direct consequence of a form of chloroquine which is incorporated into cells.

The gradual diminution in the numbers of abnormal cells after cessation of chloroquine therapy indicates that the drug effect is reversible but gives no information on the mechanisms involved. Evaluation of the reversibility of the chloroquine effect on myelocytes under in vitro conditions is not feasible, for there is ultrastructural evidence of cell degeneration after $6 \mathrm{hr}$ exposure to tissue culture conditions. ${ }^{1}$ The lifespan of circulating neutrophils and eosinophils has been shown to be relatively short (4 days) $(7,8)$. On the basis of the data reported here, the reversibility of the chloroquine effect on granulocytes within the weeks following withdrawal of the drug is likely due to replacement of the abnormal cells by a normal cell population. The presence of some abnormal granulocytes for weeks after drug withdrawal is probably related to the fact that chloro-

1 Dr. M. Fedorko, Personal observations. 
quine is stored in body depots for variable periods of time (9). On the other hand, lymphocytes, longer-lived cells (10), may either be able to reverse the cytologic effects of chloroquine or have a shortened lifespan. In the latter case there could perhaps be replacement by morphologically normal lymphocytes.

The hydrolases within lysosomes can digest either exogeneous particles taken up by the cell or endogeneous material within the cell. Previous studies have demonstrated the function of hydrolytic enzymes within specific cytoplasmic granules of neutrophils in attacking bacteria and other particles ingested by phagocytosis (2). Others have shown that lysosomes in liver and other cell types may engage in digestion of the cell's own aged or defective organelles, a process which has been termed autophagy and which probably involves the formation of digestive vacuoles (secondary lysosomes) $(11,12)$. It was probable that autophagy could occur in leukocytes, but the conditions under which the process could be induced were unknown.

Ultrastructural studies of intracellular digestion in other cell types have shown that secondary lysosomes may take the form of residual bodies consisting of membrane-bounded whorls of lamellar material (myelin figures). Some of the circulating neutrophils in peripheral blood from sarcoid patients who were treated with chloroquine showed myelin figures. From the observations on peripheral blood alone the possibility existed that the abnormal cytoplasmic granules were secondary lysosomes, perhaps representing the end stage of digestion of endogenous or exogenous material.

A study of the bone marrow leukocytes from a patient with abnormal cytoplasmic granules in mature, circulating neutrophils revealed that ultrastructural changes occur in cytoplasmic granules of immature as well as mature cells. In neutrophilic myelocytes the abnormal granules appear to be forming, for there are visible vacuolar, lamellar, and particulate components which are incorporated into cytoplasmic granules; mature neutrophils contain myelin figures.

On the basis of the findings in peripheral blood and bone marrow there are three possible cell mechanisms alone or in combination which could produce the abnormal changes: phagocytosis, an abnormality in cytoplasmic granule formation, or autophagy. The cell process which contributes to the change is likely one to which the myelocyte is most susceptible. In that case, phagocytosis could be excluded as the responsible process, since myelocytes are known not to engage in phagocytosis of exogenous material (13). On the other hand, the changes seen in early myelocytes are compatible with an effect of the drug on cytoplasmic granule formation, but no direct evidence for such an effect is available (14). The observed changes could also arise by autophagy, perhaps occurring in response to a damaging effect of the drug on some cytoplasmic component.

In other words, the abnormal cytoplasmic granules in myelocytes and mature neutrophils may represent either primary lysosomes arising from defective granule formation or secondary lysosomes resulting from an autophagic process. A meaningful answer could be obtained only if a recognizable organelle, such as mitochondrion, was found within vacuoles observed in affected myelocytes. Autophagy then could be clearly implicated as the process involved. In the specimens examined there were no recognizable cytoplasmic organelles within abnormal vacuoles or granules. In the case of the neutrophil, electron microscopic histochemistry for acid phosphatase is of little help in distinguishing between primary and secondary lysosomes.

Eosinophils from certain patients treated with chloroquine also show structural abnormalities in immature cells. Many cytoplasmic granules in very early myelocytes are normal; abnormal cytoplasmic granules become prominent as the cells mature. The more mature cells contain a marked reduction in content of the characteristics, normal cytoplasmic granule with an internal crystalloid. Instead, the cytoplasm is filled with vacuoles which contain amorphous material. The observed changes may represent an arrest in granule formation or alteration of normal cytoplasmic granules after they are formed.

Nornal lymphocytes are known not to take up exogenous material by either phagocytosis or pinocytosis. In addition, these cells normally contain few if any cytoplasmic granules. Histochemistry at the ultrastructural level helped to establish that the myelin figures which may appear in lympho- 
cytes of persons given chloroquine were positive for acid phosphatase. Autophagy is then the most reasonable explanation for the origin of these $a b-$ normal structures.

The influence of these ultrastructural changes in cytoplasmic granules on cell function is unknown. Clinically the patients were asymptomatic ; they did not report intercurrent infections, and healing from skin biopsy sites was unimpaired. Approximately one-half of the patients treated with chloroquine phosphate did not show changes in their leukocytes; the reason for this is not apparent at the present time.

The morphologic changes produced by chloroquine in leukocytes and secretory cells of other species as well as the characteristics of the chloroquine effect with in vitro cell systems will be described in subsequent communications. The ultrastructural changes described here characterize one form of acquired abnormal ("toxic") leukocyte granulation. The ultrastructure of other forms of acquired leukocyte granulation is unknown. The cell mechanisms responsible for abnormal leukocyte granulations including those in such hereditary disorders as the Chediak-Higashi syndrome and Alders anomaly remain to be investigated.

\section{References}

1. Cohn, Z. A., and J. G. Hirsch. 1960. The isolation and properties of the specific cytoplasmic granules of rabbit polymorphonuclear leucocytes. J. Exptl. Med. 112: 983.

2. Hirsch, J. G., and Z. A. Cohn. 1960. Degranulation of polymorphonuclear leucocytes following phagocytosis of microorganisms. J. Exptl. Med. 112: 1005.

3. Bäumer, A., H. Pau, and H. Conrads. 1959. Darstellung von Resochin bzw. Resochinderivaten in Geweben. Zeitschrift für Rheumaforschung. 18: 433.

4. Luft, J. 1961. Improvements in epoxy resin embedding methods. J. Biophys. Biochem. Cytol. $9: 409$.

5. Venable, J., and R. Coggeshall. 1965. A simplified lead citrate stain for use in electron microscopy. J. Cell Biol. 25 : 407.

6. Miller, F., E. de Harven, and G. E. Palade. 1966. The structure of eosinophil leucocyte granules in rodents and in man. J. Cell Biol. $31: 349$.

7. Bryant, B. J., and L. S. Kelly. 1958. Autoradiographic studies of leucocyte formation. Proc. Soc. Exptl. Biol. Med. 99 : 681.

8. Foot, E. C. 1965. Eosinophil turnover in the normal rat. Brit. J. Haematol. $11: 439$.

9. Schneider, J., A. Nenna, J. Couture. 1963. Etude comparative de la circulation dans le sang et de l'elimination urinaire de la chloroquine base et du sulfate de chloroquine. Bull. World Health Organ. 29: 417.

10. Visfeldt, J. 1964. Possible survival for years of lymphocytes in vitro without mitotic division. Acta. Pathol. Microbiol. Scand. 61 : 319.

11. Ashford, T. P., and K. R. Porter. 1962. Cytoplasmic components in hepatic cell lysosomes. J. Cell Biol. 12 : 198.

12. Swift, H., and Z. Hruban. 1964. Focal degradation as a biological process. Federation Proc. 23: 1026.

13. Jersild, M. 1948. Phagocytic activities of various types of leucocytes. Acta Med. Scand., Suppl. 131 : 238.

14. Fedorko, M. F., and J. G. Hirsch. 1966. Cytoplasmic granule formation in myelocytes. An electron microscope radioautographic study on the mechanism of formation of cytoplasmic granules in rabbit heterophilic myelocytes. J. Cell. Biol. $29: 307$. 